\title{
Development of Intelligent Clothesline Sytem
}

\author{
Lim Jia Chun ${ }^{1}$, Noor Anida Abu Talib², Haryati Jaafar ${ }^{3}$, Nordiana Shariffudin ${ }^{4}$, Nur Hidayah Ramli ${ }^{5}$ \\ \{ limjiachun_1994@hotmail.com¹, anidatalib@unimap.edu.my², haryati@unimap.edu.my ${ }^{3}$, \\ dianashariffudin@unimap.edu.my ${ }^{4}$, hidayahramli@unimap.edu.my ${ }^{5}$ \} \\ 1,2,3,4,5Faculty of Engineering Technology, Universiti Malaysia Perlis, UniCITI Alam Campus, Sg. Chuchuh, \\ 02100 Padang Besar, Perlis, Malaysia
}

\begin{abstract}
Drying clothes on a clothesline is a method that is commonly used by most of the housewives. This is because this method is very efficient with low cost needed.Nevertheless, when there is a sudden rain, the effort of clothes drying will be in vain if the housewives are not aware of it. Hence, the development of an intelligent clothesline system that would be functioning according to the weather is needed to solve this issue. In this study, light dependent resistor (LDR) and rain sensor were installed to detect the change of the environmental condition and send the input signals to the Arduino microcontroller. The characteristics of the sensors would be studied to obtain the threshold values by measuring the voltages across both sensors in different conditions. The threshold values obtained were set in the program to allow the system to determine the brightness and availability of raindrops in the surrounding. The portable prototype was designed to have a large basearea for high stability. It was also allowed to be assembled and disassembled for storagepurpose with a few simple steps needed. The completed prototype was tested outdoor ondifferent environmental conditions. The DC motor would be activated and expose theclothes when the condition was bright and not raining. Else, the clothes would be coveredunder the roof designed. The results recorded from the prototype test showed that theintelligent clothesline system developed in this project was successful as it was able todetermine the suitable condition to dry clothes.
\end{abstract}

Keywords: Microcontroller, LDR sensor, rain sensor, DC motor.

\section{Introduction}

Drying the clothes by exposing the clothesdirectly to the hot sun on a clothesline is a traditional method and is still commonlyused among housewives today. It is considered to be a simpler, low cost and mosteffective way of drying clothes. Besides, it is also proven that the ultraviolet ray fromthe sunlight could help killing available bacteria on the clothes.

Nevertheless,depression will be caused if housewives are not aware of the sudden change ofexternal environment. They would also face some difficulties to collect their clothesoutside especially when having heavy rain. In addition, most of the women would go out to work and some of them would need to go for work early in the morning. This forcing them to dry their clothes before the sun rises and would only be back at night and cause them to be fidgeting while in working place as they are worried about the possible change of weather and they could not simply go back to keep the clothes.

Hence, the development of an intelligent clothesline system is investigated and developed to solve these issues. For example, Zimal et al., (2016) proposed a clothesline system by using Atmel's AT89S52 microcontroller. With theBluetooth module installed in the system, the output of the clothesline system can becontrolled by using mobile phone with Android OS. Besides, the drying duration can beadjusted by setting the timer using the smart phone. Cutinha et al., (2016) employed the PIC16F877A microcontroller to develop the 
clothesline system. A few number of sensors such as LDR, impedance sensor, temperature sensor and rain detector sensor are used as the input switch as they can detect the change of the external environment. The LCD displays, motor and GSM module are the outputs of the system. LCD display will display the sensing value and the status of the device while the motor will rotate in both directions depending on the signal produced from the microcontroller. Subsequently, Sathish et al., (2017) used the sensors such asLDR, rain detector and temperature to detect the parameters of the 8environmental conditions.Aside than having the ability to respondaccording to the condition of the weather, the system is also powered by solar energy. Although the developments of clothesline system from the previous studies are successfully implemented, these systems are not automatically operated, costly, heavy. Therefore, there still has a room to improve such that the system is able to operate automatically, inexpensive and portable.

\section{Methodology}

The system is divided into two parts which are prototype hardware development and software development. The hardware construction of the project involves building the prototype using the chosen materials. On the other hands, the software construction is carried out by involving the programming and simulation of the circuit using Arduino IDE software.

\subsection{Hardware Development}

\subsubsection{Prototype Design Using Software}

To produce a good prototype structure, the SOLIDWORKS Premium 2016 software is used to sketch and determine the dimensions and parts assembly. If the simulation does not show good results, the designs of the parts will be reviewed and corrected until the simulation is successful.The designs of every part drawn is illustrated in Figure 1.

\subsubsection{Material Selection}

In order to choose a suitable material, a few characteristics have to be considered such as the ability to withstand heat, stiffness, strength, weight, and also the cost. In this study, polyvinyl chloride (PVC) is selected as the material of the prototype. This is due to its high stiffness, strong and is able to withstand high temperature. Besides, it is cheap and also easily available in the market. As PVC is easy to cut and join, it makes the prototype easier to build.

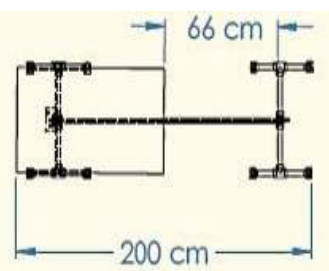

(a) Top view

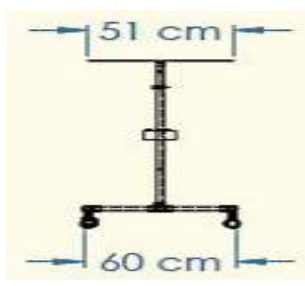

Front view (b) 


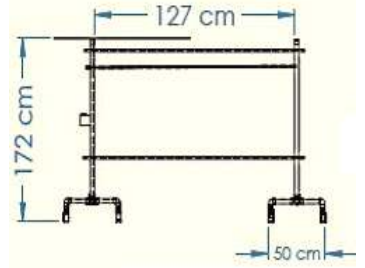

(c) Side view

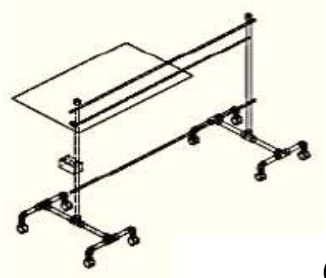

Isometric view (d)

Fig. 1. Views of prototype design from different positions

\subsubsection{Circuit Development}

In this project, the clothesline system designed will be working automatically and responding with the change of the condition of the environment. There are a few electrical components such as microcontroller, sensors and motors are used to develop the system. Figure 2 shows the workflow of the circuit operation.

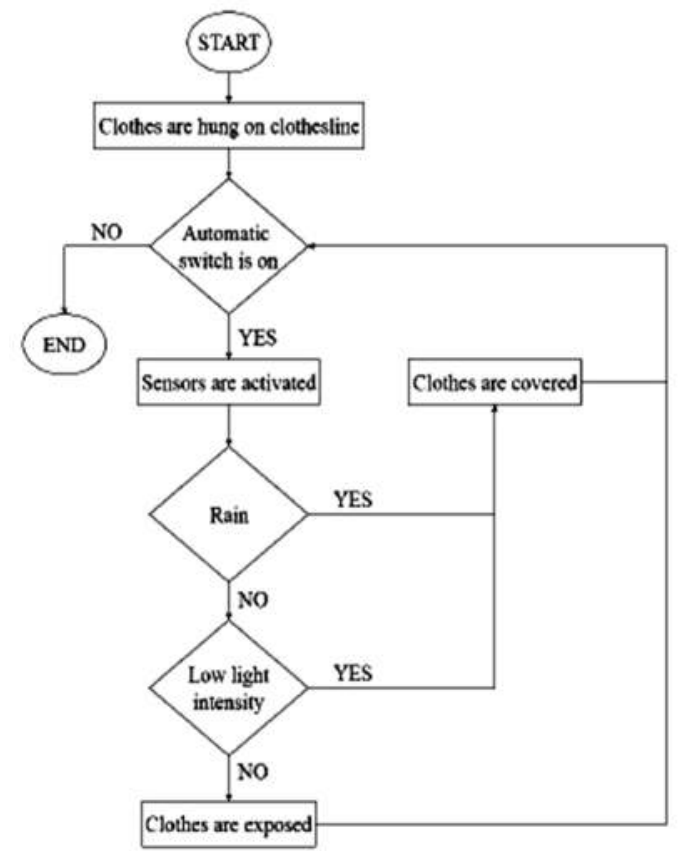

Fig. 2. Circuit operation workflow

\subsubsection{Components Selection}

It is very important to select the suitable components to run this project in order to achieve the objectives. The selection of components would need considerations based on the advantage and characteristics of the components. The power rating of each component chosen is needed to study properly to avoid bringing damage to the circuit. 
In this study, a microcontroller acts as a brain of an automation system as it could process the input signal and produce the output based on the program designed. Arduino UNO R3 is selected as the microcontroller of this project. This is due to a few advantages of it such as low cost, easy programmable and the open source library. In order to operate the system, important components such as LDR sensor, rain sensor, limit switch, DC motor and motor driver are integrated to the microcontroller.

LDR is used as switch in this project. This is due to its ability in detecting or measuring light intensity as this project requires the system to respond with the change of surrounding light intensity. Also a variable resistor, the resistance of LDR will change according to the light intensity exposes on it. As the light intensity increases, the resistance will reduce. Figure 3 shows the connection between LDR and Arduino UNO.

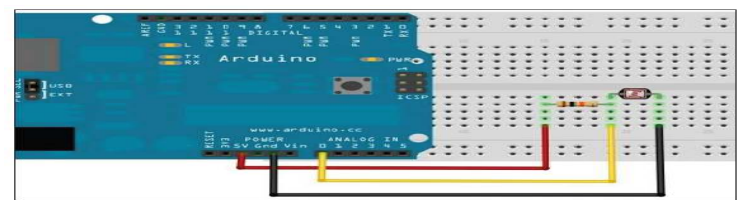

Fig. 3. Connection between LDR and Arduino Microcontroller

As raining will make the clothes wet again, a rain sensor is needed to detect the availability of raindrops. A rain sensor module is selected as it is more compatible to the Arduino microcontroller. The working principle of it is quite similar to a variable resistor. As more rain drops are detected on the sensor, its resistance will reduce. It requires a power supply between $3.3 \mathrm{~V}$ and $5 \mathrm{~V}$. Figure 4 shows how the rain sensor module is connected to the microcontroller.

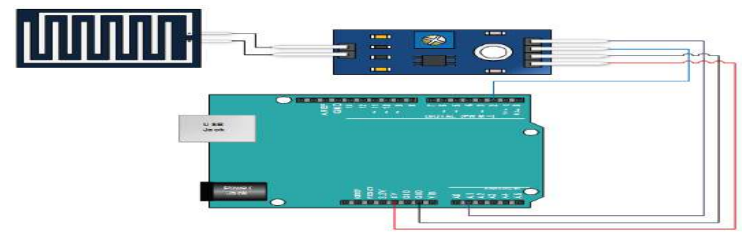

Fig.4. Connection between Rain Sensor Module and Arduino Microcontroller

Limit switch is very important in this project as it requires the motor to stop once the clothes have been pushed or pulled to a certain limit. A limit switch has 3 pins, which consist of COM, NO (Normally Open) and NC (Normally Close). If the limit switch is pressed, the circuit connection connected to NO pin will be triggered. Else, the NC pin will be remain connected. Figure 5 shows the connection between limit switch and microcontroller.

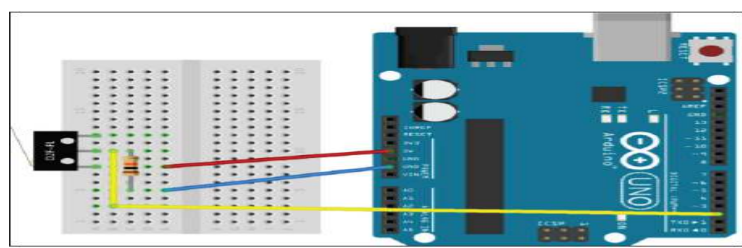

Fig. 5. Connection between Limit Switch and Arduino Microcontroller 
The DC motor is the output of the automation system in this project. The $12 \mathrm{~V} \mathrm{DC}$ motor is selected as it has a high torque up to $4 \mathrm{~kg}$ and relatively low speed. A high torque motor is required as this would make sure that the weight of multiple wet clothes can be supported. Besides, the DC motor can rotate in both direction (clockwise or anti-clockwise) based on the circuit connection.

Motor driver is used in this project as it is required to change direction of DC motor rotational motion. L293D is selected as the motor drives as it has a wide range of supplyvoltage between $4.5 \mathrm{~V}$ and $36 \mathrm{~V}$. As the DC motor used requires $12 \mathrm{~V}$, hence it is still within the voltage range.

\subsubsection{Sensor Analysis}

This process involves a few tests on the functionality of sensors according to the change of environment. In this project, two types of sensors are used, mainly LDR and Arduino rain sensor module.

The purpose of LDR test is to investigate the reaction of LDR with the change of the light intensity. To carry out the test, a voltmeter is used to measure the voltage across the LDR. In order to measure the voltage correctly, the voltmeter has to be connected parallel to the LDR. The input voltage is set to be $5 \mathrm{~V}$. The analogue voltage across the LDR will be expected to change once the light intensity changes. This experiment is carried out in a

fixed area that is exposed to sunlight, starting from 6.00 a.m. until 8.00 p.m. on 5 different days that are chosen randomly. The values are taken at every 2 hours. Figure 6 shows how the LDR test is carried out.

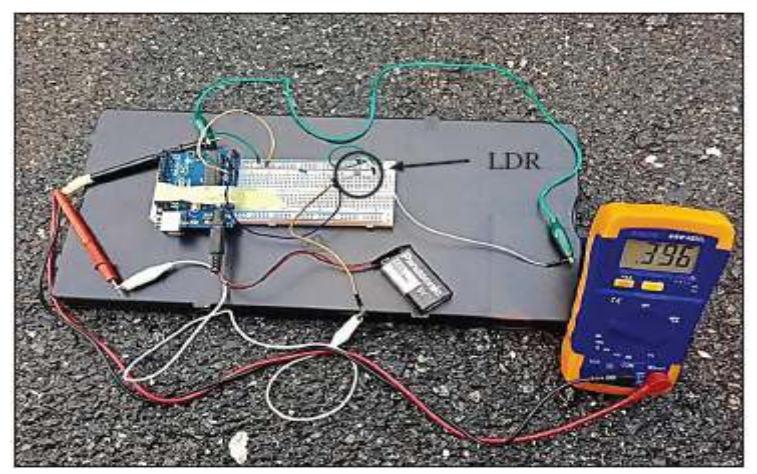

Fig. 6. LDR Test

The rain sensor module test is conducted in order to study the reaction of rain sensor module when thereis a change in volume of water fallen on it. Similar to LDR test, the voltage across therain sensor module will be measured using voltmeter. It is expected that the voltage value will change if there is a change in the volume of water. To measure the voltage across the rain sensor module, a voltmeter must be connected parallel to the sensor and the input voltage is set to 5 V.Different volumes of water $(0.0 \mathrm{ml}, 0.1 \mathrm{ml}, 0.2 \mathrm{ml}, 0.3 \mathrm{ml}, 0.4 \mathrm{ml}, 0.5 \mathrm{ml})$ are used as the manipulative variable. To place the water on the sensor plate with a desired and accurate volume, a plastic syringe is used. The test is repeated for 3 times for each volume and the average value will be calculated. Figure 7 shows how the rain sensor module test is carried out. 


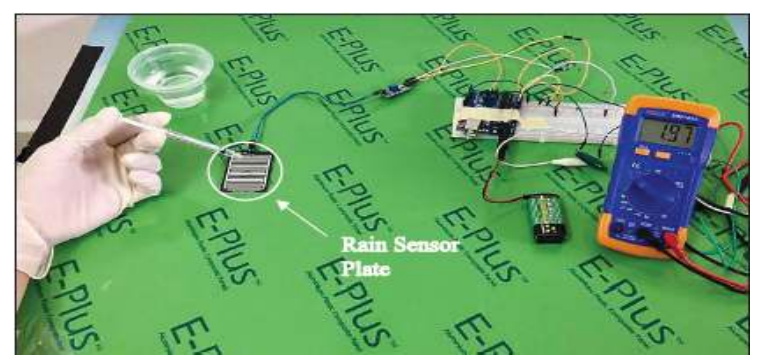

Fig. 7. Rain Sensor Module Test

\subsection{Software Development}

When the program written in Arduino IDE software is compiled with no error,simulation of the circuit will be carried out. Proteus Design Suite (Version 8.0) is chosenas the software to run this simulation. This is because the software is easy to be used with many libraries and also the output can be viewed, allowing the user to check if the coding and the circuit connection are correct.

In order to run the simulation of the circuit, the circuit has to be constructed first inside the Proteus software with all the components selected to be used in this project. While connecting the components, it is important to make sure the connection is correct to avoid errors. When the circuit is done constructing, the coding will be downloaded from Arduino IDE software and installed in the microcontroller inside the Proteus software. The simulation will be run after the download is complete and the output of the project can be viewed in animation.

\section{Experimental Results}

\subsection{Prototype Hardware Construction}

The prototype construction was completed as shown in Figure 8. It could be seen that the standing posts have a large base area, making it stable enough to stand firmly. The prototype could be easily disassembled into a few parts to reduce the area occupied for storage purpose. This would indeed help a lot in saving places. When the prototype is needed to dry clothes, the assembly process wouldtake a short time to complete with only few simple steps. Besides, it is portable and easy to move with wheels installed at the base. This allows the user to move the prototype to the desired place easily. The roof was designed to cover the clothes when it is raining.

As for the electrical hardware such as DC motor and various types of switch, their positions were also placed accordingly as shown in Figure 9. The switches consisted of emergency stop button, manual toggle switch and automatic switch button. All the switches were installed on the electrical box to ensure the housewives could operate the system conveniently. The microcontroller was kept inside the electrical box to prevent it from being exposed to rain water. The motor and limit switches were installed on the standing post to carry out the output function. As the sensors were required to obtain accurate data, the LDR and rain sensor module were placed on the roof so that they could expose to the surrounding without being covered or shaded. 


\subsection{Sensors Tests and Analysis}

\subsubsection{Light Dependent Resistor Test and Analysis}

In order to study the reaction of light dependent resistor with the condition of different time within a day, an exposed and flat area was needed to carry out this experiment to obtain data with high accuracy. Hence, Anak Bukit, Kedah, Malaysia had been chosen as the area was free from tall buildings and the sunlight could fall on the sensor without being blocked. 5 different days were randomly chosen for the experiment, starting from 6.00 a.m. until 8.00 p.m.

According to Ohm's Law, voltage is directly proportional to resistance with constant current flow (Paulus et al., 2013). As the LDR was connected with a resistor in series, the value of the voltage across the LDR would decrease if there is a rise in light intensity. This is because the resistance of LDR would decrease when the light intensity increases. The voltages across the sensor collected from Day 1 to Day 5 were recorded and tabulated as shown in Table 1.

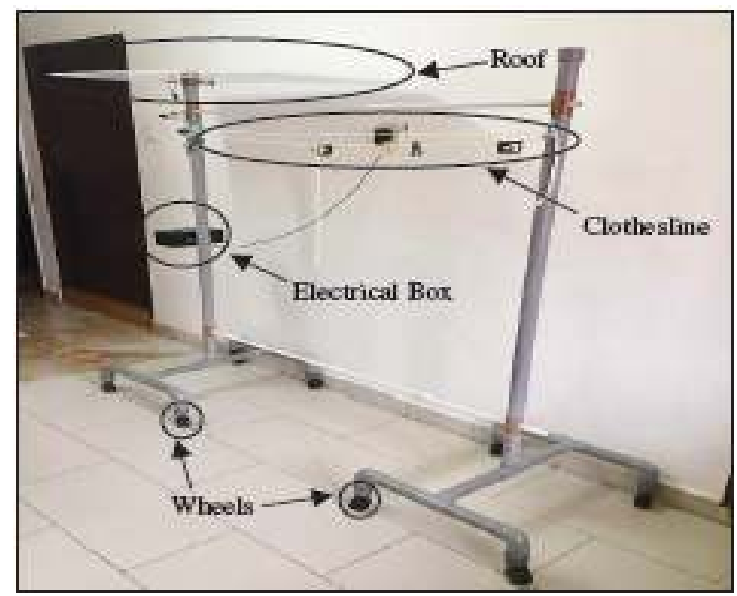

Fig. 8. Prototype (hardware)

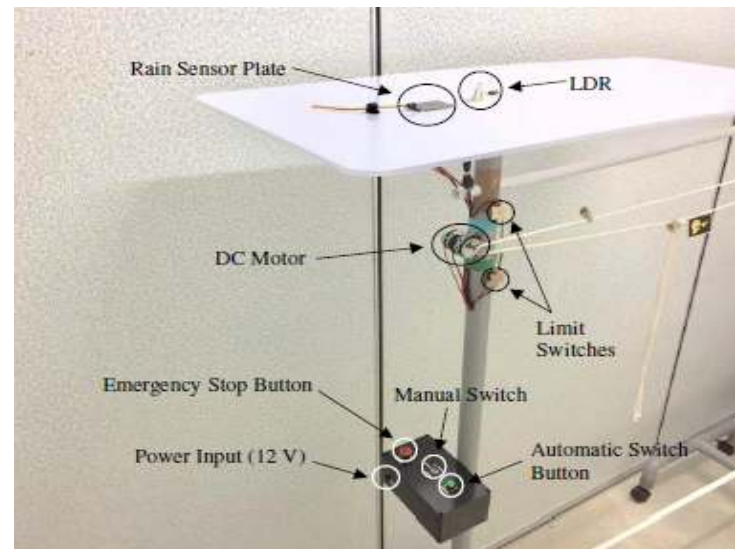

Fig. 9. Positions of electrical components and hardware 
Basically, from all the data collected, it could be concluded that the light intensity was only high with almost constant value when the time was between $0800 \mathrm{hrs}$ and $1800 \mathrm{hrs}$. This shows that the suitable hours for drying clothes is between 8.00 a.m. and 6.00 p.m.

Table 1. Voltage across LDR at Different Hours

\begin{tabular}{|l|l|l|l|l|l|}
\hline \multirow{2}{*}{ Time } & Day 1 & Day 2 & Day 3 & Day 4 & Day 5 \\
\cline { 2 - 6 } & Voltage & Voltage & Voltage & Voltage & Voltage \\
\hline 600 & 4.85 & 4.89 & 4.91 & 4.87 & 4.9 \\
\hline 800 & 0.079 & 0.081 & 0.065 & 0.76 & 0.064 \\
\hline 1000 & 0.033 & 0.035 & 0.029 & 0.81 & 0.024 \\
\hline 1200 & 0.028 & 0.026 & 0.034 & 0.75 & 0.97 \\
\hline 1400 & 0.022 & 0.021 & 0.03 & 0.63 & 0.93 \\
\hline 1600 & 0.027 & 0.03 & 0.023 & 0.094 & 0.031 \\
\hline 1800 & 0.098 & 0.1 & 0.092 & 0.103 & 0.097 \\
\hline 2000 & 4.9 & 4.91 & 4.88 & 4.92 & 4.91 \\
\hline
\end{tabular}

\subsubsection{Light Dependent Resistor Test and Analysis}

The aim of this test is to study how the voltage across the rain sensor module changes when there is a change in the water volume fallen on the surface of the sensor plate. Similar to LDR test, the rain sensor module is connected series to a resistor. As the volume of water increases, the resistance will decrease. Hence, the voltage would decrease as according to Ohm's Law. Table 2 shows the recorded voltage values across the sensor with different volumes of water applied.

Table 2. Voltage across rain sensor at different volumes of water

\begin{tabular}{|l|l|l|l|l|}
\hline Volume $(\mathrm{ml})$ & Trial 1 & Trial 2 & Trial 3 & Average \\
\cline { 2 - 5 } & Voltage & Voltage & Voltage & Voltage \\
\hline 0.0 & 4.91 & 4.90 & 4.91 & 4.91 \\
\hline 0.1 & 2.30 & 2.30 & 2.31 & 2.30 \\
\hline 0.2 & 2.19 & 2.18 & 2.20 & 2.19 \\
\hline 0.3 & 2.02 & 2.01 & 2.02 & 2.02 \\
\hline 0.4 & 1.86 & 1.86 & 1.86 & 1.86 \\
\hline 0.5 & 1.74 & 1.74 & 1.73 & 1.74 \\
\hline
\end{tabular}

Based on the data obtained from the rain sensor module test, it clearly shows that the voltage across the sensor will decrease when there is an increase of volume of water fallen on the sensor plate. When the sensor plate was dry (no water molecules present), the voltage stayed on maximum at $4.91 \mathrm{~V}$. At this moment, the resistance of the rain sensor module is considered to have reached the highest. However, when $0.1 \mathrm{ml}$ of water was placed on the sensor plate, the voltage decreased drastically until $2.30 \mathrm{~V}$. As the

volume of water was increased with an increment of $0.1 \mathrm{ml}$ each time, the voltage decreased gradually. This shows that the increasing of water volume will have effect on the resistance of the sensor.

\subsection{Digital Range Setting Calculation}

In this project, the signal from the sensors will determine the output of the system. In other words, the voltage across the sensors will be acting as the switch. As the input for the 
microcontroller is set to be analogue, it will have a range of values. However, the programming for the microcontroller requires the values to be in digital form. Hence, the average values obtained have to be converted into digital form and written in Arduino program as the threshold values for switching purpose. The values will be converted using Equation (1):

Output $=v_{\text {in }}\left(\frac{2^{n}}{v_{\text {ref }}}\right)$

It is known that $n=10$ for Arduino UNO microcontroller. As for $v_{r e f}$, the value is set to 5 $\mathrm{V}$ for both LDR and rain sensor module.

In order to determine the brightness range setting, the data collected at $1800 \mathrm{hrs}$ from the 5 -days LDR test in Table 1 is applied and the average voltage, $v_{\text {average }}$ is $0.098 \mathrm{~V}$. By substituting $v_{i n}$ with the $v_{\text {average }}=0.098 \mathrm{~V}$ in Equation (1), the digital output for LDR is 20 . Hence, the value shall be set to 20 as the threshold value for light dependent resistor. This indicates that any digital voltage value that is lesser than 20 will be considered bright and the surrounding will be considered dark if the value exceeds 20 .

On the others hand, to determine the threshold value for rain sensor module, a simple experiment is carried out. A water sprayer is used to spray the water (single spray) on the sensor plate at a height of about $30 \mathrm{~cm}$. Then, a voltmeter will be connected to the rain sensor module in parallel to measure the voltage across it. The experiment is repeated for 5 times and the average value is $3.12 \mathrm{~V}$. The average value calculated will be taken for digital conversion and set as the threshold value. By substituting $v_{i n}$ with the $v_{\text {average }}=3.12 \mathrm{~V}$ in Equation (1), the digital output for rain sensor is 657 .

\section{Conclusions}

An intelligent clothesline system had been successfully developed in this project. To fabricate an intelligent clothesline system with microcontroller, Arduino UNO R3 was selected as it is user friendly and many libraries are available in the internet due to its open source. Subsequently, the LDR sensor was used to detect the change of light intensity.Based on the results obtained, the voltage values recorded hit above $4 \mathrm{~V}$ before $6.00 \mathrm{a} . \mathrm{m}$. and after 8.00 p.m. as the surrounding was dark at those moment. As for rain sensor, it was used to detect the raindrops. When the voltage across the rain sensor module decreases, it shows that water droplets are present on the sensor plate. According to the results, the voltage decreased linearly as the water volume increased. Based on the calculations made for range setting, the threshold values for both LDR and rain sensor module were set as 20 and 657 respectively after digital conversion. The threshold values would discriminate the voltage values to determine the brightness of the surrounding and the availability of raindrops. This also means that any digital voltage value which is lesser than 20 across the LDR would be considered as bright. Else, the surrounding would be known as dark. As for rain sensor module, any voltage value across it that is higher than 657 would be considered as no rain. If the value is lesser than 657 , the surrounding is known to be raining.

\section{Acknowledgements}

The authors would like to express their gratitude for the financial support provided by Universiti Malaysia Perlis Short Term Grant (9001-00526). 


\section{References}

[1] Zimal, S.,Khodaskar, S., Kusalkar, M.,Khairnar, V., Mumbai, N., 2016. Android Smart Life,vol. 4, no. 2, pp. 181-183.

[2] Cutinha, L. S.,Pai, V.,Sadhana, B., 2016. Automatic Cloth Retriever System, pp.243246, 2016.

[3] Sathish, K.,Selvaganapathy, M., Siva, S.,Kumaresan, G., 2017. Design andExperimental Study on Automatic Cloth Retrieval and Drying System, vol. 3, pp. 49-57.

[4] Paulus W.,Opitz, A., 2013. Clinical Neurophysiology Ohm's law and tDCS over the centuries, vol. 124, pp. 429-430. 